\title{
Caracterización preliminar del cultivo de arracacha Arracacia xanthorrhiza Bancroft en el departamento de Boyacá
}

\author{
Preliminary characterization of arracacha Arracacia xanthorrhiza \\ Bancroft crop in the department of Boyaca
}

\section{Astrid Lorena Muñozi; Alvaro Alvarado G.2; Pedro José Almanza-Merchán³.}

1 I. A., M.Sc., semillero grupo GIPSO, Universidad Pedagógica y Tecnológica de Colombia, Tunja. Colombia. astrid.munoz@ uptc.edu.co

2 Docente Asociado. I. A. M.Sc. Universidad Pedagógica y Tecnológica de Colombia, Tunja. Colombia, Tunja. Colombia. alvaro.alvarado@uptc.edu.co

3 Docente asociado, I. A. Ph.D. en Ciencias Agropecuarias, Universidad Pedagógica y Tecnológica de Colombia, Tunja. Colombia. pedro.almanza@uptc.edu.co

Fecha recepción: Noviembre 23 de 2014

Fecha aceptación: Abril 13 de 2015

\section{RESUMEN}

Los estudios realizados en torno a la diversidad genética de la arracacha en Colombia y en el departamento de Boyacá son escasos. En los municipios productores del departamento de Boyacá, se realizó la identificación preliminar de los materiales genéticos existentes, se visitaron fincas de productores, con el objetivo de documentar sus saberes en torno a la especie y otros aspectos del sistema productivo. La metodología empleada es de tipo cualitativo - descriptivo, basada en criterios no métricos de recolección de datos, dependiendo de las características de la investigación y del criterio del investigador. Se recolectaron muestras de material vegetal, en el cual se observaron los siguientes caracteres: color del follaje, color del tallo, color y presencia del anillo en la raíz. Se pudo determinar que los tres colores predominantes del follaje identificados corresponden a: verde, verde-violáceo y morado. En el tallo se observó: verde y morado-rojizo. La raíz presentó coloración blanca o amarilla, con vasos de aceite de colores blancos o morados. Se pudo determinar la existencia de seis materiales genéticos denominados Yema de Huevo, Paliverde, Palirrusia, Palinegra, Sata y Sata morada.

Palabras clave: Raíces andinas, materiales genéticos, agrobiodiversidad. 


\section{ABSTRACT}

In the department of Boyaca, and in Colombia in general, studies on the genetic diversity of arracacha are scarce. A preliminary identification of the existing genetic material in the producing municipalities of the department of Boyaca was conducted. Visits to production farms were made in order to document the knowledge regarding the species and other aspects of the production system. A qualitativedescriptive methodology was used, based on non-metric criteria for data collection, depending on the research characteristics and the researcher's criteria. Plant material samples were collected and the following traits were recorded: leaf color, stem color, color and presence of the root ring. Three main leaf colors were determined: green, purple-green, and purple. Regarding the stem, green and reddish-purple were observed. The root showed a white or yellow color, with white or purple colored oil vessels. The existence of six genetic materials was determined, which were named Huevo, Paliverde, Palirrusia, Palinegra, Sata, and Satamorada.

Keywords: Andean roots, genetic material, agrobiodiversity.

\section{INTRODUCCIÓN}

La arracacha A. xanthorrhiza Bancroft, es una especie de la familia umbelífera, originaria de los Andes septentrionales y domesticada en el continente americano, desde hace unos 3000 años, siendo cultivada y consumida por los campesinos andinos desde la época pre-Inca (Alvarado y Ochoa, 2010A). Jiménez (2005) menciona que ésta planta es originaria de Jamaica, sin embargo, se cree que es originaria de los andes del norte de Sudamérica, por encontrase en esta zona la mayoría de especies de éste género.

Según Domínguez et al. (2006) se encuentra distribuida desde la República Bolivariana de Venezuela hasta Bolivia, en un rango altitudinal que va de los 1000 a $3000 \mathrm{msnm}$. Actualmente, se cultiva en el Brasil, América Central, el Caribe, Estados Unidos y Australia, lo cual demuestra, no sólo su capacidad de adaptación a zonas agroecológicas diferentes y a diversos mercados, lo que implica un creciente interés y demanda por este tubérculo. A pesar de considerarse durante muchos años como un cultivo de valor económico potencial y con posibilidades de expansión, su biología es poco conocida (Knudsen et al., 2004).

En el departamento de Boyacá, para el año de 2011, reportó una superficie sembrada en arracacha de 870 ha, con una producción de 7.206 toneladas con un rendimiento de $10.930 \mathrm{kgha}^{-1}$ (MADR, 2011). Dentro de los municipios productores de esta especie, se encuentran: Ciénega, Jenesano, La Capilla, Somondoco, Tibaná, Pachavita y Garagoa. Sin embargo, la determinación de las características del material vegetal producido aún no ha sido identificada y caracterizada plenamente. Por esta razón, el objetivo de este trabajo buscó la identificación y descripción morfológica preliminar de los materiales genéticos, que se utilizan en los sistemas de producción de los principales municipios productores de arracacha en el departamento de Boyacá, como son: Tibaná, Ciénega, Jenesano, Pachavita, Garagoa, La Capilla y Somondoco. 
Igualmente, se estudiaron los componentes involucrados en el sistema de producción para determinar el impacto sobre la economía de las veredas productoras y en la calidad de vida de las familias campesinas, lo cual permitirá corroborar, unificar y ampliar el conocimiento técnico existente de esta especie.

\section{MATERIALES Y MÉTODOS}

Al no contarse con información preliminar sobre el tamaño o características de la población objetivo, se partió de una investigación de tipo cualitativo, por lo tanto, se utilizó un tipo de muestreo no probabilístico, también llamado muestra dirigido o intencional. La elección de los elementos no depende de la probabilidad sino de las condiciones que permiten hacer el muestreo, como acceso o disponibilidad, conveniencia, criterios del investigador, entre otros (Scharager, 2001).

En este tipo de muestreo, no es posible calcular con precisión el error estándar de estimación, es decir, no se puede determinar el nivel de confianza con que se hace dicha estimación, razón por lo cual, no todos los productores de arracacha en cada municipio tuvieron la misma probabilidad de ser seleccionados, por lo que es esperable la no representatividad de todos los miembros de la población. La selección de los elementos de la muestra (productores), se realizó en el transcurso de los recorridos en campo. A cada uno se aplicó una entrevista semiestructurada, basada en una guía de preguntas en donde el entrevistador tiene la libertad de adicionar otras preguntas, para precisar conceptos u obtener mayor información (Hernández et al., 2007).
Los procesos de investigación, se basaron en la observación directa, la obtención de registros escritos, fotográficos y de la experiencia del productor, con una metodología de tipo cualitativo descriptivo (Alvarado y Ochoa, 2010B); por lo tanto, se aplicó un muestreo de tipo no probabilístico. Se identificaron veredas, fincas y agricultores dedicados a este cultivo, mediante la metodología productor a productor.

Se aplicó la encuesta participativa, en la cual se recolectaron los datos facilitados por los productores a través de un cuestionario con la información relacionada con el sistema de producción de arracacha, como: subsistema suelos (fertilización, frecuencia, análisis de suelos), cultivo (área, siembra, realización de prácticas culturales, semilla, procedencia, distancia de siembra, cantidad, cultivo solo o asociado), enfermedades, plagas, malezas (manejo fitosanitario) y un intercambio de ideas y experiencias. La unidad de muestreo, la constituyo cada finca, donde se encuentra establecido el cultivo de arracacha.

Para la caracterización preliminar e identificación de los materiales se tuvo en cuenta algunas de las características morfológicas, que son fácilmente identificables por los productores, haciendo uso de los descriptores elaborados para dicha actividad como color de hoja, tallos, raíz, forma de la raíz, presencia de anillos en la raíz, nombre regional.

Se aplicaron 70 encuestas, con un promedio de ocho encuestas por municipio, dependiendo de las características de producción. La información colectada con respecto a los caracteres cuantitativos fue almacenada y analizada con base en la herramienta de datos de Excel. 


\section{RESULTADOS Y DISCUSIÓN}

En la Tabla 1, se muestra la caracterización preliminar, basada en las particularidades morfológicas más comunes y de fácil identificación por los productores, de seis variedades identificadas en la zona de estudio y que han sido denominadas regionalmente por los productores como: Sata, Sata Morada, Paliverde, Palirrusia Garagoeña, Palinegra y Yema de Huevo Cajamarca. En estos materiales geneticos, se encuentran diferencias en el color de hojas, tallos y en la forma y color de la raíz. En estudios similares en otras zonas, se coincide con la presente investigación, al mencionarse que las bases para diferenciarlas, es el color del follaje, de la raíz y especialmente por la presencia o ausencia de un anillo de color morado en la raíz (Seminario, 2004; Vásquez et al., 2004; Alvarado y Ochoa, 2010A). El rango de altura donde se cultiva arracacha en los municipios del departamento de Boyacá está entre 1140 y 2427 msnm. Según Alvarado y Ochoa (2010B) en el municipio de Gachantiva, Sata Morada y Sata son cultivados entre los 2380 y 2427 msnm; Paliverde, Palirrusia, Palinegra, Yema de Huevo, yucatana, de tarro blanca y de Tarro Amarilla, se encuentran cultivadas entre los 2000 a $2600 \mathrm{msnm}$.
De acuerdo con Higuita (1977), en Colombia se conocen tres formas hortícolas de arracacha, conocidas como: Amarilla (raíces amarillas, follaje verde); Blanca (raíces blancas, follaje verde) y Morada (raíces amarillas, follaje carmín). Estas tres formas básicas han dado lugar a nueve diferentes formas hortícolas, resultantes de la combinación de color de la raíz y del follaje. Por su parte, Hodge (1949) indica que los indios Quillacingas del Putumayo, cultivan 11 tipos de arracacha reconocidas por nombres locales (Seminario, 2004).

Teniendo en cuenta la forma y la estructura de la raíz, los agricultores de la región, clasifican dos tipos: los denominados "Tarro" (Sata y Sata Morada), caracterizados por presentar la raíz principal gruesa, y las llamadas "Apios", cuyas raíces son numerosas y pequeñas (Paliverde, Palirrusia Garagoeña, Palinegra y Yema de Huevo Cajamarca). Alvarado y Ochoa (2010B) mencionan que las variedades conocidas regionalmente, como Apios, corresponden a cultivares que fueron introducidos de la zona de Cajamarca (Tolima), en la década de los años 50. Higuita y Rodríguez (1975) reportaron la existencia, en el Centro de Investigaciones de Tibaitatá (Cundinamarca), de una colección de

Tabla 1. Caracterización cualitativa de los materiales genéticos de A. xanthorriza en el departamento de Boyacá.

\begin{tabular}{lllccc}
\hline \multicolumn{1}{c}{ NOMBRE REGIONAL } & \multicolumn{1}{c}{$\begin{array}{c}\text { COLOR DE } \\
\text { LA HOJA }\end{array}$} & $\begin{array}{c}\text { COLOR DEL } \\
\text { TALLO }\end{array}$ & $\begin{array}{c}\text { COLOR DE } \\
\text { LA RAİZ }\end{array}$ & $\begin{array}{c}\text { FORMA DE } \\
\text { LA RAIZ }\end{array}$ & $\begin{array}{c}\text { PRESENCIA } \\
\text { ANILLO EN } \\
\text { LA RAİZ }\end{array}$ \\
\hline Sata & Verde & Verde & Blanco & Tarro & $\mathrm{Si}$ \\
Sata morada & Verde- morado & Morado & Blanco & Tarro & $\mathrm{Si}$ \\
Paliverde & Verde & Verde & Amarillo & Apio & $\mathrm{Si}$ \\
Palirrusia-garagoeña & Verde & Morado suave & Amarillo & Apio & No \\
Palinegra & Verde- morado & Morado & Amarillo & Apio & No \\
Yema de Huevo- Cajamarca & Verde & Verde & Amarillo & Apio & No \\
\hline
\end{tabular}


15 formas hortícolas, que podrían denominarse variedades, procedentes de Perú, Putumayo (Valle de Sibundoy) y Antioquia, agrupadas en tres clases: Blancas: Salamineña (Antioquia), Amarillas: Yema de Huevo (Ibagué) y otras; Moradas. Según Higuita (1968), estas diferentes formas hortícolas, se reconocen por el color de follaje (verde o morado), el color externo de la raíz (blanca, crema, amarilla o morada) y color de los vasos (anillos) de aceite de la raíz (gris, morado, violeta o incoloro).

De acuerdo con la caracterización cualitativa, se determinó la preferencia del agricultor por municipio, por un tipo de arracacha (Tabla 2), factor en el cual incide la demanda del producto. Según Alvarado y Ochoa (2010B) Paliverde, Palirrusia, Palinegra y Yema de Huevo se encuentran reportadas como las más cultivadas y consumidas en los municipios de Boyacá, en tanto que Sata y Sata morada, fueron identificados en el municipio de Gachantivá.

Sistema de producción. La unidad agrícola familiar predominante en los municipios visitados, corresponde al tipo mini-microfundista, donde el $80 \%$ corresponden a predios menores de 3 ha, de los cuales el $60 \%$, corresponden a lotes entre
1600 y $3200 \mathrm{~m}^{2}$. Como se tratan de sistemas de economía de pequeña escala, estos se caracterizan porque los agricultores, no aplican ningún tipo de tecnología, al no recibir asistencia técnica, y al considerarse como un cultivo tradicional sin el uso de insumos agrícolas. El 10\%, de las fincas cultivan áreas que van desde los $3200 \mathrm{~m}^{2}$ hasta las 14 ha, perteneciendo estas últimas a agricultores que poseen capacidad económica para atender los requerimientos agronómicos propios del cultivo.

De acuerdo con la información obtenida de los agricultores de municipios productores de arracacha en el departamento de Boyacá, no se tiene una época definida, en la que se programen la siembra de la planta. Amaya y Julca (2006) mencionan que la arracacha es una planta rústica que puede cultivarse durante todo el año, por lo que el agricultor puede manejarla de acuerdo con la demanda del producto, orientado a la obtención de mejores precios. En cuanto a la distancia de siembra, varía según criterio del productor, en promedio se encontró que el $80 \%$, manejan distancias entre plantas de $0,2 \times 1 \mathrm{~m}$, con densidades poblacionales de 50.000 plantas ha ${ }^{-1}$, y el $20 \%$ utilizan distancias de siembra de $0,20 \times 0,40$ $\mathrm{m}$, para una densidad de 125.000 plantas $\mathrm{ha}^{-1}$.

Tabla 2. Incidencia del cultivo de A. xanthorhiza en los municipios productores del departamento de Boyacá.

\begin{tabular}{ll}
\hline \multicolumn{1}{c}{ MUNICIPIO } & \multicolumn{1}{c}{ VARIEDADES COMUNES } \\
\hline Gachantivá & Sata y Sata morada \\
Tibaná & Palirrusia, Paliverde, Palinegra y Yema de Huevo \\
Ciénega & Palirrusia, Paliverde, Palinegra y Yema de Huevo \\
Jenesano & Paliverde, Palinegra y Yema de Huevo \\
Pachavita & Palirrusia, Paliverde y Yema de Huevo \\
Garagoa & Palirrusia y Yema de Huevo \\
Capilla & Palirrusia, Yema de Huevo y Palinegra \\
Somondoco & Palirrusia, Yema de Huevo y Palinegra \\
\hline
\end{tabular}


En el trabajo realizado por González y Guerra (2010) se reportan distancias de siembra de 1,0 $x 1,0$ m donde se siembran hasta 10.000 plantas ha $^{-1}$. Alvarado y Ochoa, (2010A) mencionan que para el municipio de Cajamarca (Tolima) las distancias de siembra óptimas son $0,5 \times 1 \mathrm{~m}$, con una densidad de 20.000 plantas ha-1 ${ }^{-1}$ Alvarado y Ochoa (2010B), reportan rendimientos en los municipios del departamento de Boyacá que oscilan entre 9 y $12 \mathrm{t} \mathrm{ha}^{-1} \mathrm{y}$ en Colombia entre 10,9 y $1,4 \mathrm{t} \mathrm{ha}^{-1}$.

Respecto al uso de mano de obra, para la ejecución de las principales labores de cultivo, estas son realizadas en un $47,54 \%$ por el núcleo familiar, en fincas pequeñas, asociadas a cultivos pequeños y que no demandan grandes esfuerzos. En un $44,26 \%$, se contratan obreros que se encargan de labores que requiera el cultivo y el 8,19\% restante, emplean obreros y al mismo tiempo se apoya de la mano de obra familiar disponible, para repartir labores y de algún modo disminuir costos de producción, su explotación se concentra en manos de pequeños productores, quienes por décadas han desarrollado tecnologías de cultivo propias. El argumento coincide con el de Espinal y Martínez (2003), quienes mencionan que el predominio en los regímenes productivos de pequeñas explotaciones son carentes de tecnología y en terrenos no aptos para la mecanización, donde el $97 \%$ del total de agricultores siembran menos de 10 ha.

La fertilización se realiza de acuerdo con la disponibilidad de dinero para la compra de insumos. En algunos casos se reportan entre tres a cuatro aplicaciones durante el ciclo del cultivo. Una primera al momento de siembra, cuando se presenta el periodo de lluvias y durante el desyerbe. Normalmente, se aplican hasta 3 t.ha-1 de materia orgánica (pollinaza sin descomponer) y entre 400 a $500 \mathrm{Kg} \cdot \mathrm{ha}^{-1}$ de fertilizante químico. La mayoría de los productores (32\%) utilizan fertilizantes químicos como 15-15-15; además, en un $60 \%$ de los productores aplican abonos orgánicos, que se obtienen de la misma finca, manifestando en su totalidad la falta de asistencia técnica, en tanto que el 28,6\% utilizan fuentes de fertilizantes químicos mezclados con fuentes orgánicas y se aprovecha la fertilización de los cultivos cuando se siembra en asocio. En el momento de la siembra, es común la aplicación de enmiendas calcáreas desde $100 \mathrm{~kg}$ en 6,400 $\mathrm{m}^{2}$ a $0,5 \mathrm{t} \mathrm{ha}^{-1}$.

El 80\% de los encuestados no reportan problemas causados por organismos fitopatógenos en su cultivo; sin embargo, el 20\% mencionan, que el cultivo presenta una pudrición causada por el hongo Sclerotinia sclerotiorum, siendo la variedad de tipo Apio, la más afectada. Posiblemente, y de acuerdo con Alvarado y Ochoa (2010A), está asociada al exceso de humedad en el suelo. Son varios los métodos reportados por los productores, entre los cuales se destaca la erradicación de la planta afectada, para evitar que sea foco de esclerocios.

Aunque no representan un problema limitante las malezas en este cultivo, debido a su abundante follaje que compite con la maleza que crece a su lado. Sin embargo, los productores siempre están pendientes dando un manejo oportuno en sus primeros estadios de desarrollo. El control de malezas de forma manual, se realiza mediante 2 a 3 deshierbes al año cada 2 o 3 meses, según la agresividad de la maleza en el cultivo.

De acuerdo con la información suministrada por los encuestados, en el material Paliverde se reportaron rendimientos entre 2000 a 10.000 
kg.ha-1 ${ }^{-1}$ Esta variedad se analiza desde diferentes factores, teniendo en cuenta tipos de suelos, fertilización, área de producción, sistema productivo (asociado o no) y condiciones climáticas entre otras. Transcurridos los 12 a 18 meses, en caso de los materiales genéticos Palirrusia y Palinegra, los agricultores proceden a cosechar la arracacha, completando su madurez a consideración de ellos. En el caso de Yema de Huevo, la cosecha se hace entre los 6 a 12 meses después de siembra; esta diferencia se ve marcada por condiciones fenológicas (variedades tempranas o tardías) y la influencia del clima, especialmente temperatura. En este sentido, de acuerdo con Seminario (2004), la fecha de inicio de la cosecha de una plantación, puede variar con la ecología del lugar y el cultivar, así que la plasticidad de los cultivares actuales permite tener lapsos de cosecha que varían de 4 a 12 meses. Seminario y Coronel (2004) indican que en Mollebamba (Cajamarca, Perú) la cosecha se inicia entre los 8 y 12 meses según el cultivar. El indicador de cosecha más importante es la edad de la planta, pero también el campesino hace muestreos para ver el tamaño y grosor de las raíces; si el tamaño de raíces no es adecuado, se espera unos meses más.

Los productores destinan la producción tanto para la comercialización como para el autoconsumo. Los principales mercados o sitios de venta se encuentran en los municipios de Jenesano, Ramiriquí. En el mercadeo juega un rol importante los intermediaros, los cuales se encargan de la comercialización en las centrales de abastos de Bogotá y Tunja, y en algunos casos en el mismo mercado municipal. Como caso especial, se encontró que la comercialización de la producción del municipio de Gachantivá, el principal sitio de venta es Moniquirá, principalmente en los restaurantes, pues es allí donde se preparan sus conocidas picadas con arracacha morada. Igualmente, en los mercados o plazas de cada municipio se puede encontrar puntos de venta del producto y hace parte del consumo de la familia campesina y destinada para la alimentación de los hogares campesinos.

\section{CONCLUSIONES}

Se identificaron las variedades denominadas por los productores como: Sata, Sata morada, Paliverde, Palirrusia garagoeña, Palinegra y Yema de Huevo cajamarca, que presentan diferencias en el color de hojas, tallos y en la forma y color de la raíz.

El cultivo de arracacha en los principales municipios productores del departamento de Boyacá, es poco tecnificado, tradicional en las familias caracterizado por su rusticidad y poca demanda de insumos, considerándose como un cultivo limpio, libre de aplicaciones de plaguicidas.

El consumidor prefiere cultivares del tipo de raíz Apio, que es una variedad que por su sabor y presentación es la más sembrada en la zona productora de Boyacá.

\section{AGRADECIMIENTOS}

Los autores agradecen el apoyo del Grupo de Investigación en Desarrollo y Producción Agraria Sostenible (GIPSO) de la Universidad Pedagógica y Tecnológica de Colombia y el apoyo económico y administrativo de la dirección de investigaciones DIN, quienes apoyaron la realización de la investigación, Código SGI 895, convocatoria DIN 002-2011 apoyo a proyectos internos capital semilla. 


\section{BIBLIOGRAFIA}

ALVARADO, A. y L. OCHOA, 2010A. Cultivo de arracacha (A. xanthorrhiza Bancroft) en los municipios de Turmequé y Boyacá (Boyacá, Colombia). imprenta Universidad Pedagógica y Tecnológica de Colombia. Tunja. I. 97 p.

ALVARADO, A. y L. OCHOA, 2010B. Tecnologías locales de producción de arracacha (A. xanthorrhiza Bancroft) en el municipio de Boyacá, departamento de Boyacá. En revista U.D.C.A. Actualidad y Divulgación Científica. 13(1):23 p.

AMAYA, J. y JULCA, J. 2006. Arracacha (Arracacia xanthorrhiza Bancroft). Gerencia Regional de Recursos Naturales y Conservación del Medio Ambiente. Gobierno Regional La Libertad. Perú. $15 \mathrm{p}$.

DOMINGUEZ. S., DELGADO. R., CORONADO. D. y DOS SANTOS. F. 2006. Desarrollo rural desde las personas, Una experiencia del mejoramiento de la competitividad de la arracacha. Primera edición. Red de Agroindustria Rural del Perú. Lima Perú. 116 p.

ESPINAL, C. y MARTÍNEZ, H. 2003. La Cadena de papa en Cundinamarca frente a las negociaciones comerciales hemisféricas. Corporación Latinoamericana Misión Rural, Gobernación de Cundinamarca. Dirección de Planeación. Bogotá. 188 p.

GONZÁLEZ, F. y GUERRA, D. 2010. Evaluación de la producción y residuos de cosecha en dos variedades comerciales de arracacha en Boyacá, Boyacá. Trabajo de grado. Programa de Ingeniería Agronómica Universidad Pedagógica Tecnológica de Colombia. Tunja. 38 p.
GRINELL, R.M. 1977. Social work research evaluation: Quantitative and Cualitative approaches. Quinta edición, Itasca, Illinois: E. E. Peacock Publisher.123 p.

GUERRA D, F. GONZÁLEZ y A. ALVARADO. 2012. Producción y residuos en dos materiales comerciales de arracacha (Arracacia xhanthorriza Banc) en Boyacá. Revista Ciencia y Agricultura. 9(2):77 - 86.

HERNÁNDEZ,R.,FERNÁNDEZ,C.yBAPTISTA, P. 2007. Metodología de la Investigación. Cuarta edición. McGraw-Hill. 850 p.

HIGUITA, F. 1977. La horticultura en Colombia. Manual de asistencia técnica $N^{0} 5$. Segunda edición. Instituto Colombiano Agropecuario. $37-41 \mathrm{p}$.

HIGUITA. F. y RODRÍGUEZ. E. 1975. El cultivo de la Arracacha. Revista Esso Agrícola. Volumen XXI. (4):10 - 11.

HIGUITA, F. 1968. El cultivo de la arracacha en la Sabana de Bogotá. Separata Agricultura Tropical. 14(3):139 - 147.

HODGE, W.H. 1949. La arracacha comestible. Revista Facultad Nacional de Agronomía 10:232 - 254.

JIMENEZ, F. 2005. Características Nutricionales de la Arracacha (Arracacia xanthorrhiza) y sus perspectivas en la alimentación. Publicación virtual red peruana de alimentación y nutrición. Lima, Perú. 22p. En: http://www.rpan. org/monografias/monografia002.pdf, consulta: enero, 2015.

REVISTA DE CIENCIAS AGRÍCOLAS Enero - Junio 2015, 32 (1): 3 - 11 
KNUDSEN, S., HERMANN, M., DOS SANTOS, F. y SORENSEN, M. 2004. Inducción de floración en el cultivo de arracacha (Arracacia xanthorrhiza Bancroft). RAICES ANDINAS Contribuciones al conocimiento y a la capacitación. 6:197 - 213 p.

MINISTERIO DE AGRICULTURA Y DESARROLLO RURAL (MADR). 2011. Evaluaciones Agropecuarias Municipales 2011. Corporación Colombiana Internacional (CCI), Secretaría de Fomento Agropecuario, Dirección de Desarrollo Agropecuario, Grupo de Información y Planificación Agropecuaria, Unidad Regional de Planeación Agropecuaria (URPA). 61 p.

SCHARAGER, J. 2001. Muestreo probabilístico. Metodología de la investigación para las ciencias sociales. Pontificia Universidad Católica de Chile. Santiago de Chile. 3 p.

SEMINARIO, J. 2004. Origen de las Raíces Andinas. 376p. En: Seminario, J. (ed.). Raíces Andinas: Contribuciones al conocimiento y a la capacitación. Serie: Conservación y uso de la biodiversidad de raíces y tubérculos andinos: Una década de investigación para el desarrollo (1993-2003) No. 6. Universidad Nacional de Cajamarca, Centro Internacional de la Papa, Agencia Suiza para el Desarrollo y la Cooperación. Lima, Perú.

SEMINARIO, J. y CORONEL T. 2004. Aspectos etnobotánicos y económicos de la arracacha en Mollebamba,Huambos (19). En:J.Seminario(ed.). Raíces Andinas: Contribuciones al conocimiento y la capacitación. Serie: Conservación y uso de la biodiversidad de raíces y tubérculos andinos: Una década de investigaciones para el desarrollo (1993-2003) No 6. Universidad Nacional de Cajamarca, Centro Internacional de la Papa, Agencia Suiza para el Desarrollo y la Cooperación. Lima, Perú. 261 - 277 p.
VÁSQUEZ, N., MEDINA, C. y LOBO, M. 2004. Caracterización morfológica de la colección colombiana (Tolima, Huila, Boyacá, Cauca) de arracacha (Arracacia xanthorrhiza). p 165-178. En: Seminario, J. (ed.). Raíces Andinas: Contribuciones al conocimiento y a la capacitación. Serie: Conservación y uso de la biodiversidad de raíces y tubérculos andinos: Una década de investigación para el desarrollo (1993-2003) No. 6. Universidad Nacional de Cajamarca, Centro Internacional de la Papa, Agencia Suiza para el Desarrollo y la Cooperación. Lima, Perú. 